\title{
Upregulation of adiponectin receptor 1 and 2 mRNA and protein in adipose tissue and adipocytes in insulin-resistant women with polycystic ovary syndrome
}

\author{
B. K. Tan • J. Chen $\cdot$ J. E. Digby $\cdot$ S. D. Keay • \\ C. R. Kennedy $\cdot$ H. S. Randeva
}

Received: 23 May 2006 / Accepted: 24 July 2006 / Published online: 26 September 2006

(C) Springer-Verlag 2006

\begin{abstract}
Aims/hypothesis Polycystic ovary syndrome (PCOS) is a multifaceted metabolic disease linked with insulin resistance (IR) and obesity. Adiponectin, which is lower in IR states, exerts its glucose-lowering and anti-inflammatory effects by activating two receptors, ADIPOR1 and ADIPOR2. There are no data on the relative expression of these receptors in adipose tissue of PCOS women.

Methods We investigated the expression of adiponectin receptors from corresponding s.c. and omental (o.m.) adipose tissue in women with PCOS compared with matched non-PCOS women. As there is a disturbance in the steroid milieu in PCOS women, we also assessed the effects of testosterone and oestradiol on adiponectin receptors using adipocytes and adipocyte explants. Realtime RT-PCR and western blotting were used to assess the relative adiponectin receptor mRNA expression and protein
\end{abstract}

B. K. Tan and J. Chen contributed equally to this work.

Electronic supplementary material Supplementary material is available for this article at http://dx.doi.org/10.1007/s00125-006$0419-9$ and is accessible to authorised users.

B. K. Tan · J. Chen · J. E. Digby $\cdot$ S. D. Keay $\cdot$

H. S. Randeva $(\square)$

Endocrinology and Metabolism Group,

Clinical Sciences Research Institute, Warwick Medical School,

University of Warwick,

Coventry CV4 7AL, UK

e-mail: hrandeva@bio.warwick.ac.uk

S. D. Keay $\cdot$ C. R. Kennedy

Centre for Reproductive Medicine,

University Hospitals Coventry and Warwickshire NHS Trust,

Coventry, UK production, respectively. Biochemical measurements were performed in our hospital's laboratory.

Results We are the first to describe adiponectin receptor expression and production, in corresponding s.c. and o.m. human adipose tissues at the mRNA and protein level. We demonstrate the upregulation of mRNA expression and protein production of adiponectin receptors in women with PCOS, in s.c. and o.m. adipose tissue. Treatment of adipose tissue explants and adipocytes with testosterone and oestradiol induced the expression of adiponectin receptor mRNA and protein. There was a significant positive association between ADIPOR1/R2 expression and homeostasis model assessment, testosterone, oestradiol and triglycerides and a negative relationship with sex hormonebinding globulin.

Conclusions/interpretation The precise reason for the upregulation of adiponectin receptors seen in PCOS women, a pro-diabetic state, is unknown, but it appears that sex steroids may play a role in their regulation in adipose tissue.

Keywords Adipocytes · Adiponectin receptors .

Adipose tissue $\cdot$ Insulin resistance .

Polycystic ovary syndrome · Sex steroids

\begin{tabular}{ll}
\multicolumn{2}{l}{ Abbreviations } \\
ADIPOR1 & adiponectin receptor-1 \\
ADIPOR2 & adiponectin receptor-2 \\
E2 & $17 \beta$-oestradiol \\
FAI & free androgen index \\
HOMA & homeostasis model assessment \\
IR & insulin resistance \\
o.m. & omental
\end{tabular}


PCOS polycystic ovary syndrome

SHBG sex hormone-binding globulin

\section{Introduction}

Polycystic ovary syndrome (PCOS) is characterised by menstrual dysregulation and hyperandrogenism, and is associated with hyperinsulinaemia [1]; it is a multifaceted metabolic disease with dyslipidaemia, overt type 2 diabetes mellitus, insulin resistance (IR) and visceral obesity being more prevalent than in weight-matched control subjects $[1,2]$.

Adiponectin, which is lower in insulin-resistant states [3], orchestrates its glucose-lowering and anti-inflammatory effects by activating two membrane receptors, adiponectin receptor-1 (ADIPOR1) and adiponectin receptor-2 (ADIPOR2) [4]. These receptors mediate paracrine effects of adiponectin in adipose tissue [5].

Therefore, given that PCOS is a pro-diabetic and pro-inflammatory state [6], with a higher prevalence of obesity [6], we sought to investigate the expression of adiponectin receptors from adipose tissue, both s.c. and omental (o.m.), in women with PCOS compared with matched non-PCOS women. As there is a disturbance in the steroid milieu in PCOS women, we assessed the effects of sex steroids on adiponectin receptors in adipose tissue and adipocytes.

\section{Subjects and methods}

After an overnight fast, blood samples and abdominal s.c. and o.m. adipose tissue were obtained $(08.00-10.00 \mathrm{~h})$ from adult female patients undergoing elective surgery for infertility investigation. All samples were obtained during the early follicular phase (days 2-4 from the first day of spontaneous bleeding episode); serum/plasma were immediately aliquoted on ice and stored at $-80^{\circ} \mathrm{C}$ (see Electronic supplementary material [ESM]). The control group had no discernible cause for infertility (unexplained infertility). Equal numbers of women $(n=8)$ were recruited in each group, one that consisted of women with PCOS and the other healthy control subjects (ESM Table 1). The local research ethics committee approved the study and all patients involved gave their informed consent, in accordance with the guidelines in The Declaration of Helsinki 2000.

Our PCOS women met all three diagnostic criteria as per the revised 2003 Rotterdam ESHRE/ASRM PCOS Consensus Workshop Group ([7]; see ESM).

Biochemical and hormonal analysis Assays for covariates (ESM Table 1), were performed using an automated analyser (Abbott Architect; Abbott Laboratories, Abbott Park, IL, USA). Adiponectin was measured by commercial RIA (Linco Research, St Charles, MO, USA). The intraassay variation was $5.7 \%$. The estimate of IR by homeostasis model assessment (HOMA) score was calculated as previously described [8].

Primary explant culture Adipose tissue organ explants were cultured using methods described by Fried and Moustaid-Moussa ([9]; see ESM).

Isolation of primary adipocytes Adipocytes were isolated as previously described [10]. Briefly, adipose tissue was exposed to collagenase digestion (Hanks' Balanced Salt Solution containing $3 \mathrm{mg} / \mathrm{ml}$ collagenase [type II] and $1.5 \%$ BSA) in a shaking water bath at $37^{\circ} \mathrm{C}$ for $1 \mathrm{~h}$. Mature adipocytes were separated from the stromal vascular cells through an inert oil, bis(3,5,5 trimethylhexyl) phthalate (Fluka Chemicals, Gillingham, UK).

Total RNA extraction and cDNA synthesis Total RNA was extracted and reverse transcribed as previously described [11].

RT-PCR Protocol conditions and quantitative PCR of ADIPOR 1 and ADIPOR 2 were performed on a Roche Light Cycler system (Roche Molecular Biochemicals, Mannheim, Germany), as previously described ([11]; see ESM).

Western blotting Protein lysates were prepared by homogenising adipose tissue in modified radioimmunoprecipitation lysis buffer (Upstate, Lake Placid, NY, USA) according to the manufacturer's instructions, and subjected to western blotting protocol conditions and analysis as previously described by us ([11]; see ESM).

Immunohistocytochemistry Adipose tissue sections from subjects were cut at $3 \mu \mathrm{m}$ and floated onto 3-aminopropyltriethoxy-silane coated slides and immunohistocytochemical analysis was performed as previously described ([12]; see ESM).

Statistical analysis Non-parametric tests were used. Differences between two groups were assessed using the MannWhitney $U$ test. Data involving more than two groups were assessed by Kruskal-Wallis and Friedman's ANOVA. For western immunoblotting experiments, the densities were measured using a scanning densitometer coupled to scanning software (ImageQuant; Molecular Dynamics, Amersham Pharmacia, Little Chalfont, UK). Spearman rank correlation was used for calculation of associations between variables. Values are expressed as means \pm SEM unless indicated otherwise. $p<0.05$ was considered significant for all analyses. 


\section{Results}

Clinical, hormonal and metabolic features of subjects are described in ESM Table 1.

Expression of adiponectin receptor genes in normal and PCOS women There was no significant difference in $A D I P O R 1 / R 2$ mRNA in either fat depot in normal or PCOS women (Fig. 1a,b). However, there was significant upregulation of $A D I P O R 1$ and $A D I P O R 2$ in both fat depots of
PCOS women (Fig. 1a,b; $p<0.05$ ). Furthermore, significantly higher expression of $A D I P O R 1$ and $A D I P O R 2$ was noted in isolated s.c. adipocytes in PCOS women (Fig. 1c,d; $p<0.05)$.

Protein production of adiponectin receptors in normal and PCOS women Western blotting detected significantly greater ADIPOR1 (42 kDa) protein in both s.c. and o.m. adipose tissue of PCOS women (Fig. 1e; $p<0.01$ ). However, ADIPOR2 (35 kDa) protein was significantly upregulated
Fig. 1 a, b mRNA expression of ADIPOR1 (a) and ADIPOR2

(b) in human s.c. and o.m. adipose tissues of PCOS and control subjects $(n=8)$, using real-time RT-PCR. Data are shown as means \pm SEM. ${ }^{*} p<0.05$ vs control subjects. c, d mRNA expression of ADIPORI (c) and ADIPOR2 (d) in isolated s.c. adipocytes from PCOS and control subjects $(n=8)$, using real-time RT-PCR. $* p<0.05$ vs control subjects. The PCR products obtained were $125 \mathrm{bp}$ for $A D I P O R 1$ and $190 \mathrm{bp}$ for ADIPOR2. As a negative control for all the reactions, preparations lacking reverse transcriptase were used. RNAs were assayed from three independent biological replicates. e, f Western blot analysis of membrane protein extracts from control subjects and PCOS adipose tissue $(n=8)$ demonstrate that the antibody against ADIPOR1/R2 recognised bands with apparent molecular masses of $42 \mathrm{kDa}$ and $35 \mathrm{kDa}$, respectively, in both s.c. and o.m. depots (inserts). Densitometric analysis of the immune complexes revealed that there is a significant increase in protein expression of ADIPOR1 in both s.c. and o.m. of PCOS women when compared with control subjects $(* * p<0.01)$, whereas a significant increase in protein expression of ADIPOR2 was observed only in s.c. but not o.m. of PCOS women when compared with control subjects $\left({ }^{*} p<0.05\right)$. $P S L$, phospho-stimulated light units a

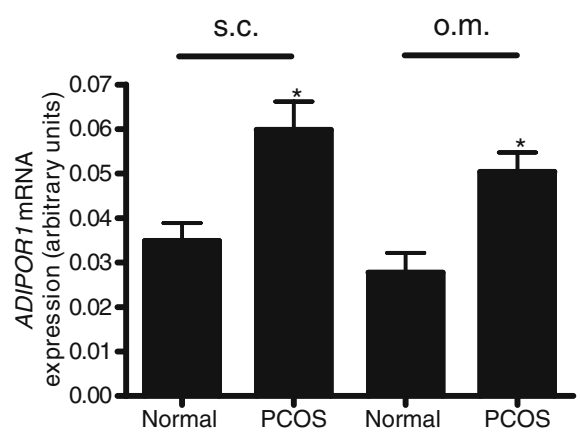

C
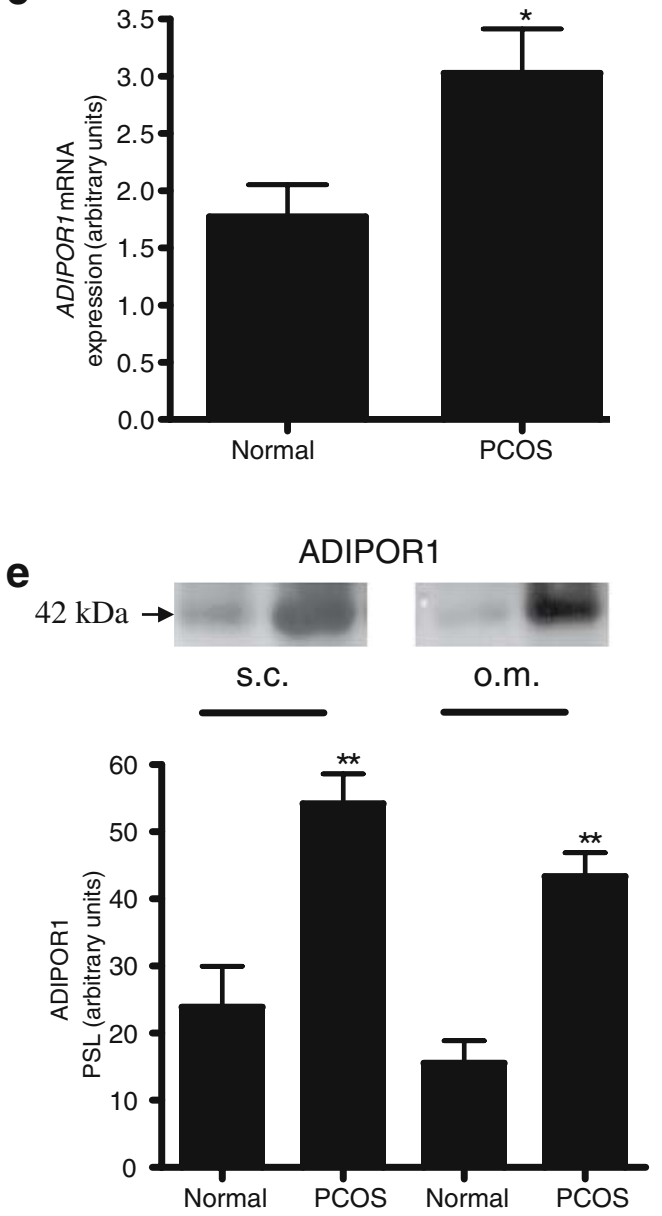

b
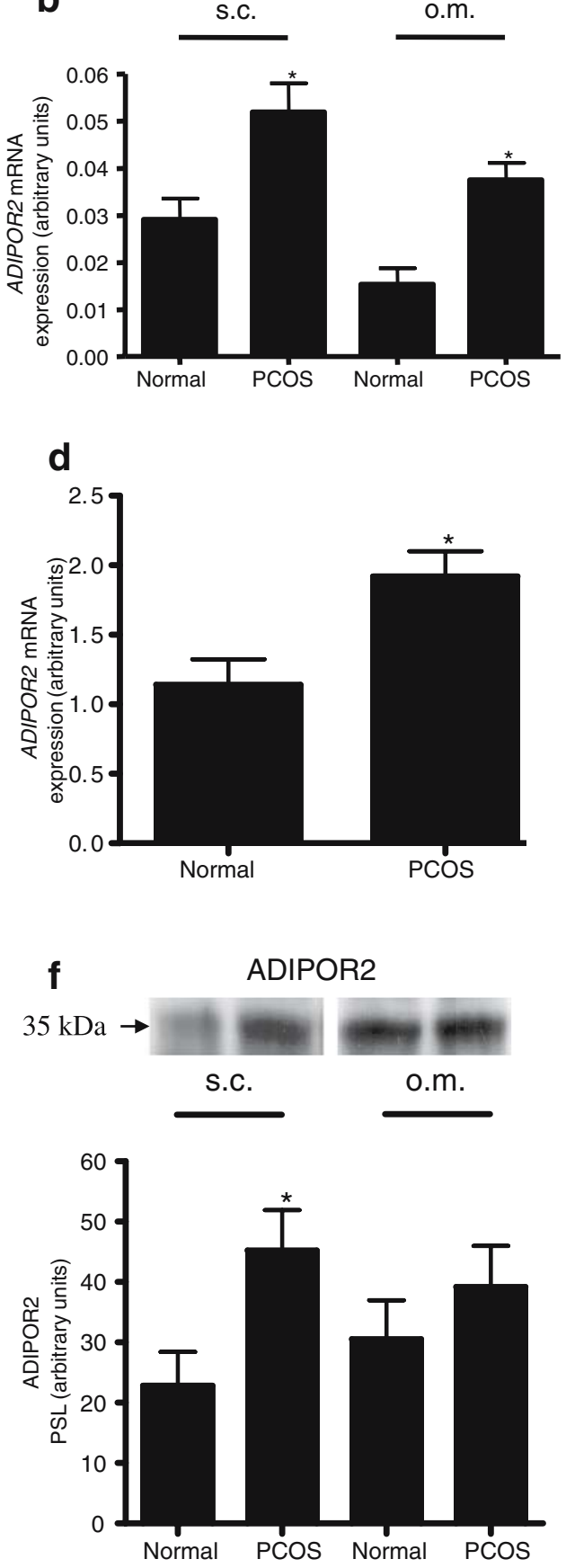
only in s.c. adipose tissue of PCOS women (Fig. 1f; $p<0.05)$. Immunohistocytochemical analysis revealed intense membrane staining for both ADIPOR1 and ADIPOR2 in adipocytes (ESM Fig. 1).

Effects of testosterone and 17 $\beta$-oestradiol (E2) on adiponectin receptor expression and production in isolated human s.c. adipocytes and adipose tissue explants Human s.c. adipocytes treated with testosterone and E2 had significantly increased ADIPOR1 (testosterone: $p<0.01$; E2: $p<0.05$ ) and ADIPOR2 expression (testosterone: $p<0.05$; E2: $p<0.01$; Fig. $2 \mathrm{a}, \mathrm{b})$. The effects of testosterone on $A D I P O R 1 / R 2$ expression were similar in s.c. adipose tissue explants, although with respect to testosterone, ADIPOR 1 mRNA was significantly elevated but the increase in $A D I P O R 2$ mRNA failed to reach significance (testosterone: $p=\mathrm{NS}$; E2: $p<0.05$; Fig. $2 \mathrm{c}, \mathrm{d}$ ).
Importantly, these mRNA findings were supported by protein data utilising western immunoblotting: ADIPOR1 (testosterone: $p<0.01$; E2: $p<0.01$ ) and ADIPOR2 (testosterone: $p<0.01$; E2: $p<0.01$; Fig. $2 \mathrm{e}, \mathrm{f}$ ). Experiments were performed on s.c. adipose tissue, given technical restrictions of obtaining adequate o.m. tissue during laparoscopy.

Correlation of adiponectin receptor gene expression with covariates In s.c. adipose tissue, ADIPOR1 expression was positively associated with insulin $(r=0.84, p=0.009)$, HOMA $(r=0.89, p=0.002)$, E2 $(r=0.81, p=0.016)$, testosterone $(r=0.84, p=0.009)$, the free androgen index (FAI; (testosterone $[\mathrm{nmol} / 1] / \mathrm{SHBG}[\mathrm{nmol}]) \times 100)(r=0.83$, $p=0.01)$, triglycerides $(r=0.74, p=0.036)$ and negatively with sex hormone-binding globulin (SHBG) $(r=-0.75$, $p=0.034)$; however, ADIPOR 2 correlated only with HOMA $(r=0.70, p=0.05)$ and E2 $(r=0.72, p=0.044)$.
Fig. 2 a, b Effects of testosterone and E2 $100 \mathrm{nmol} / 1$ on ADIPOR1 and ADIPOR2 mRNA in human s.c. primary adipocytes at $4 \mathrm{~h}$ were assessed by real-time RT-PCR, as compared with basal (no supplement); ${ }^{*} p<0.05,{ }^{* *} p<0.001$. c, d Effects of testosterone and E2 $(100 \mathrm{nmol} / \mathrm{l})$ on ADIPOR1 and $A D I P O R 2$ mRNA in human s.c. adipose tissue explants at $4 \mathrm{~h}$ were assessed by real-time RT-PCR, as compared with basal (no supplement); ${ }^{*} p<0.05$, $*^{* *} p<0.001$. e, f Effects of testosterone and E2 $(100 \mathrm{nmol} / \mathrm{l})$ on ADIPOR1 and ADIPOR2 protein production as assessed by western blotting in human s.c. adipose tissue explants at $24 \mathrm{~h}$. Densitometric analysis of the immune complexes revealed that there was a significant increase in protein production of ADIPOR1/R2 with both testosterone and E2 when compared with basal (no supplement); ${ }^{*} p<0.05,{ }^{* *} p<0.001$. Values are means \pm SEM of six experiments. $P S L$, phospho-stimulated light units
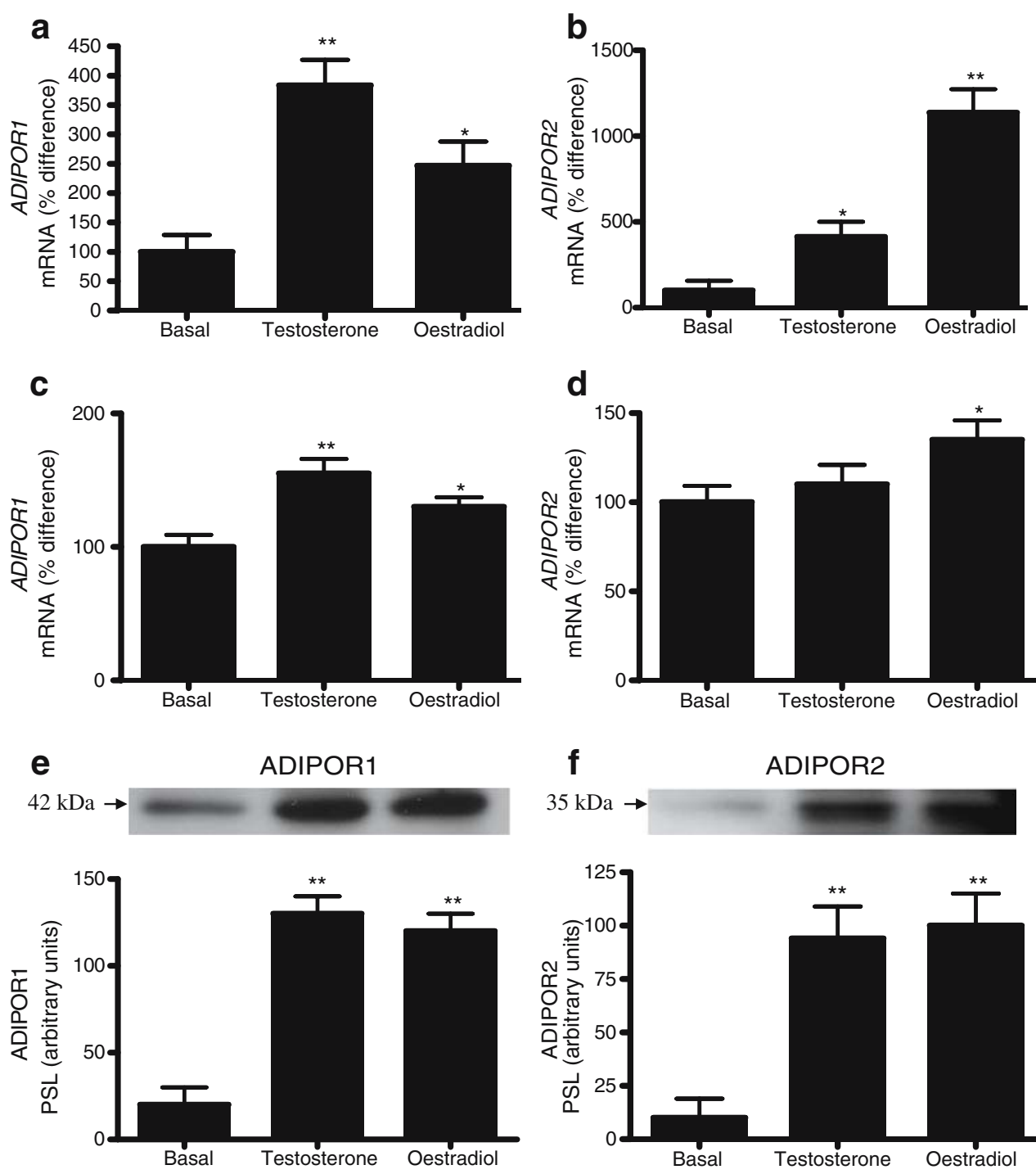
In o.m. adipose tissue, $A D I P O R 1$ expression was associated with insulin $(r=0.86, p=0.006)$, HOMA $(r=0.82, p=0.012)$, E2 $(r=0.85, p=0.008)$, testosterone $(r=0.89, p=0.002)$, FAI $(r=0.93, p=0.001)$ and triglycerides $(r=0.84, p=0.009)$ and negatively with SHBG $(r=-0.79$, $p=0.018)$. Unlike s.c. adipose tissue, in o.m. adipose tissue ADIPOR 2 expression was associated with insulin $(r=0.88$, $p=0.004)$, HOMA $(r=0.91, p=0.002)$, testosterone $(r=0.84$, $p=0.009)$, and FAI $(r=0.92, p=0.001)$ and negatively with SHBG $(r=-0.81, p=0.014)$.

\section{Discussion}

In this short communication, we describe for the first time the expression of adiponectin receptor-1 and -2 in corresponding s.c. and o.m. human adipose tissues at both the mRNA and protein level. Importantly, we present novel data showing the presence and upregulation of ADIPOR1/R2 in both these adipose tissue depots in PCOS women, and the effect of testosterone and E2 on these receptors, these women having a relative excess of these steroids. The precise reasons for our findings need to be determined.

The upregulation of adiponectin receptors in adipose tissue of PCOS women, in whom diabetes is more prevalent, may reflect an increased need for adiponectin receptor signalling and may sensitise the adipocytes to the effects of adiponectin, including its lipolytic activity. Tan et al. [5] showed that as adiponectin receptor mRNA concentrations increased, in vivo postprandial adipose tissue clearance of triglycerides increased. In our study, PCOS women had higher triglyceride levels. It is possible that the increased expression of adiponectin receptors in PCOS women may modulate triglyceride clearance.

Human preadipocytes are capable of active androgen generation [13]. Moreover, adipose tissue aromatises testosterone to E2 locally, which may be pertinent given the hyperandrogenic state in PCOS women. Sex steroids improve insulin sensitivity and decrease risk of diabetes [14]; however, the precise mechanisms are unclear. Interestingly, we noted significant positive associations between sex steroids and both adiponectin receptors. Furthermore, we provide novel evidence that testosterone and E2 increase adiponectin receptor- 1 and -2 mRNA and protein. Therefore, the increased expression and production of adiponectin receptors in PCOS women, with no significant difference in BMI, WHR and plasma adiponectin concentrations compared with control subjects, may be a consequence of the hyperandrogenic state, given the significantly raised testosterone and FAI in PCOS women. However, it is unknown whether these sex steroid effects on adiponectin receptors occur in other tissues.
A limitation of our study may relate to the number of subjects. However, obtaining BMI/WHR-matched and menstrual cycle-synchronised blood and tissue samples impeded subject recruitment. Not withstanding, our observations are highly consistent and significant, and raise interesting questions on the mechanisms regulating adiponectin receptor expression. Moreover, although we present novel data showing that sex steroids influence adiponectin receptor mRNA expression and protein production in adipose tissue, some of our covariate correlations may not have a causative significance, and indeed result from the fact that PCOS is characterised by hyperandrogenism.

In conclusion, we present novel data with increased adiponectin receptors in PCOS adipocytes, and their regulation by sex steroids. The precise mechanisms for our findings remain to be elucidated, but the upregulation of adiponectin receptors may sensitise adipocytes to the effects of adiponectin, and act as a compensatory mechanism by which insulin sensitivity is at least partially restored in these insulin-resistant PCOS women. Future studies, with larger numbers of subjects, should give a better insight into the molecular mechanisms of adiponectin and its receptors in insulin-resistant PCOS women.

Acknowledgements This study was funded by The General Charities of the City of Coventry. We are grateful to E. Karteris for proofreading the manuscript.

Duality of interest All authors have nothing to disclose, and there is no duality of interest.

\section{References}

1. Dunaif A (1997) Insulin resistance and the polycystic ovary syndrome: mechanisms and implications for pathogenesis. Endocr Rev 18:774-800

2. Rajkhowa M, Bichnell J, Jones M et al (1994) Insulin sensitivity in women with polycystic ovary syndrome: relationship to hyperandrogenemia. Fertil Steril 61:605-612

3. Matsuzawa Y (2006) The metabolic syndrome and adipocytokines. FEBS Lett 580:2917-2921

4. Yamauchi T, Kamon J, Ito Y et al (2003) Cloning of adiponectin receptors that mediate antidiabetic metabolic effects. Nature 423:762-769

5. Tan GD, Debard C, Funahashi T et al (2005) Changes in adiponectin receptor expression in muscle and adipose tissue of type 2 diabetic patients during rosiglitazone therapy. Diabetologia 48:1585-1589

6. Legro RS (2003) Polycystic ovary syndrome and cardiovascular disease: a premature association? Endocr Rev 24:302-312

7. Fauser B (2004) Revised 2003 consensus on diagnostic criteria and long-term health risks related to polycystic ovary syndrome (PCOS). Hum Reprod 19:41-47

8. Matthews DR, Hosker JP, Rudenski AS et al (1985) Homeostasis model assessment: insulin resistance and beta-cell function from fasting plasma glucose and insulin concentrations in man. Diabetologia 28:412-419 
9. Fried SK, Moustaid-Moussa N (2001) Culture of adipose tissue and isolated adipocytes. Methods Mol Biol 155:197-212

10. Rodbell M (1964) Metabolism of isolated fat cells. Effects of hormones on glucose metabolism and lipolysis. J Biol Chem 239:375-380

11. Chen J, Tan B, Karteris E et al (2006) Secretion of adiponectin by human placenta: differential modulation of adiponectin and its receptors by cytokines. Diabetologia 49:1292-1302

12. Randeva HS, Karteris E, Grammatopoulos D et al (2001) Expression of orexin-A and functional orexin type 2 receptors in the human adult adrenals: implications for adrenal function and energy homeostasis. J Clin Endocrinol Metab 86:4808-4813

13. Quinkler M, Sinha B, Tomlinson JW et al (2004) Androgen generation in adipose tissue in women with simple obesity-a site-specific role for 17 beta-hydroxysteroid dehydrogenase type 5. J Endocrinol 183:331-342

14. Margolis KL, Bonds DE, Rodabough RJ et al (2004) Effect of oestrogen plus progestin on the incidence of diabetes in postmenopausal women: results from the Women's Health Initiative Hormone Trial. Diabetologia 47:1175-1187 